\title{
Quantum enhancement of $N$-photon phase sensitivity by interferometric addition of down-converted photon pairs to weak coherent light
}

\author{
Takafumi Ono and Holger F. Hofmann \\ Graduate School of Advanced Sciences of Matter, Hiroshima University, Kagamiyama \\ 1-3-1, Higashi Hiroshima 739-8530, Japan
}

\begin{abstract}
It is shown that the addition of down-converted photon pairs to coherent laser light enhances the $N$-photon phase sensitivity due to the quantum interference between components of the same total photon number. Since most of the photons originate from the coherent laser light, this method of obtaining non-classical $N$-photon states is much more efficient than methods based entirely on parametrically downconverted photons. Specifically, it is possible to achieve an optimal phase sensitivity of about $\delta \phi^{2}=1 / N^{3 / 2}$, equal to the geometric mean of the standard quantum limit and the Heisenberg limit, when the average number of down-converted photons contributing to the $N$-photon state approaches $\sqrt{N / 2}$.

PACS numbers: 42.50.Dv 42.50.St 03.67.-a 42.50.Lc

E-mail: h.hofmann@osa.org
\end{abstract}




\section{Introduction}

Recent advances in the generation and control of non-classical multi-photon states have made it possible to realize super-sensitive phase measurements, where quantum correlations between the photons reduce the errors in a phase measurement below the standard quantum limit valid for uncorrelated photons [1]. Up to now, many of the theoretical and experimental efforts have focused on the generation of path entangled states (also known as NOON states), where the photons are in a superposition of all photons in one path or all photons in the other path [2, 3, 4, 5, 6, 1, 8, 9, 10, 11, 12, 13, 14]. However, experimental noise and low post-selection efficiencies have made it difficult to overcome the standard quantum limit at photon numbers higher than two [15]. It is therefore useful to consider alternative approaches that do not require maximal path entanglemed input states [16, 17, 18, 19, 20, 21, 22]. In particular, these proposals make more direct use of the standard source of non-classical light, the emission of photon pairs by spontaneous parametric down-conversion. It might also be worth noting that the first experiment to actually beat the standard quantum limit with a four photon state [21] was based on one of these proposals [17] and used the four photon component $|2 ; 2\rangle$ of two mode down-conversion as input state.

Yet another alternative approach towards achieving optical interferometry beating the standard quantum limit is the use of squeezed states [23, 24, 25, 26, 27, 28, 29, 30]. These states are usually characterized by a continuous parameter describing the gradual suppression of quantum noise observed in the output of the interferometer. Squeezing can thus describe the transition from weak to strong non-classical effects within the same conceptual framework. It has already been known for a long time that improved phase sensitivity can be achieved by using a squeezed vacuum input in the empty port of a two path interferometer [23]. However, the improvement of phase sensitivity is then only equal to the quadrature squeezing in the input. The recent breakthroughs in

phase sensitive measurements based on path entanglement show that such high levels of quadrature squeezing are not necessary to achieve greatly improved phase sensitivities. Instead, it is sufficient to use spontaneous parametric down-conversion, which is formally equivalent to a squeezed vacuum with extremely low squeezing levels. The improvement of phase sensitivity then originates from multi-photon quantum interferences in the $N$ photon component detected in the output. It may thus be possible to achieve high levels of phase squeezing in $N$-photon states by using multi-photon quantum interference effects instead of quadrature squeezing, in closer analogy to the methods employed to generate maximally path entangled states.

In this paper, we investigate the possibility of gradually squeezing the $N$-photon component of a weak coherent light input from a single mode laser by interferometrically adding photon pairs from a much weaker parametric down-conversion. It is shown that the quantum interference between the generation of photon pairs in the laser and the generation of down-converted photon pairs results in $N$-photon squeezing, where the squeezing parameter is given by $N$ times the ratio of the pair generation amplitude $\gamma$ 
and the squared amplitude of the coherent light $\alpha^{2}$. Since $\gamma$ is the quadrature squeezing parameter of the down-converted light, the selection of the $N$-photon component in coincidence counting thus amplifies the squeezing by a factor of $N / \alpha^{2}$. It is therefore possible to increase the squeezing level obtained by reducing the amplitude of the coherent input light. We find that this kind of squeezing can achieve a maximal phase sensitivity of about $\delta \phi^{2}=1 / N^{3 / 2}$ when the average number of down-converted photons contributing to the $N$-photon state approaches $\sqrt{N / 2}$. Even at this optimal squeezing value, most of the photons originate from the coherent laser light. Therefore, high photon numbers $N$ can be obtained even without particularly bright down-conversion sources. The interferometric addition of down converted photon pairs to coherent laser light thus provides an extremely efficient tool for beating the standard quantum limit at high photon numbers.

The rest of the paper is organized as follows. In section 2 we review the quantum mechanics of optical phase estimation and derive a definition of phase squeezing based on the Hilbert space representation of uncertainties. In section 3 , we show how destructive interference between the uncertainties of two quantum state components can be used to achieve this kind of phase squeezing. In section 4, we use operator relations to derive the general squeezing characteristics obtained by quantum interference between laser light and down-converted photon pairs. It is shown that the amount of squeezing in the $N$-photon component depends on a single parmeter, $\eta=N \gamma / \alpha^{2}$. Significant amounts of squeezing can therefore be obtained if $N \gg \alpha^{2}$. In section 5, the limits of interferometric squeezing are considered. It is shown that this kind of squeezing can reduce the phase error to $\delta \phi^{2}=1 / N^{3 / 2}$, the geometric mean of the standard quantum limit and the Heisenberg limit. In section 6, we describe the squeezed state in the input photon number basis and analyze the efficiency of the $N$-photon state generation. It is shown that the probability of generating an $N$-photon state is orders of magnitude higher than a corresponding pair state generation with two mode down-conversion. In section 7, we illustrate the effects of squeezing by presenting numerical results for the eight photon case. The relation between classical field interference and quantum interference effects is illustrated by graphs showing the photon number statistics of the eight photon interference fringe. In section 8 , the results are summarized and conclusions are presented.

\section{Phase measurement and squeezing}

To illustrate the quantum mechanics of N-photon interference, it is useful to consider a conventional Mach-Zehnder interferometer, as shown in fig. 1. The light enters the interferometer in the input modes $a$ and $b$ described by the corresponding annihilation operators $\hat{a}$ and $\hat{b}$. The two modes then mix at the input beam splitter BS1, so that each path inside the interferometer is represented by an equal superposition of $\hat{a}$ and $\hat{b}$. Inside the interferometer, each path experiences a different phase shift. However, only the phase difference between the two paths is relevant for the observed interference. In 
the following, we assume that the phase difference is $\phi-\pi / 2$, so that, at $\phi=0$, the output modes obtained after the path modes interfer at the output beam splitter BS2 are also equal superpositions of $\hat{a}$ and $\hat{b}$.

As indicated in fig,1, we can now introduce the Schwinger representation of the two mode photon statistics by identifying $\hat{J}_{1}$ with half the photon number difference between the input modes, $\hat{J}_{2}$ with half the photon number difference between the output modes at $\phi=0$, and $\hat{J}_{3}$ with half the photon number difference between the two paths inside the interferometer. In terms of the input modes $\hat{a}$ and $\hat{b}$, the operators of the Schwinger representation read

$$
\begin{aligned}
& \hat{J}_{1}=\frac{1}{2}\left(\hat{a}^{\dagger} \hat{a}-\hat{b}^{\dagger} \hat{b}\right) \\
& \hat{J}_{2}=\frac{1}{2}\left(\hat{a}^{\dagger} \hat{b}+\hat{a} \hat{b}^{\dagger}\right) \\
& \hat{J}_{3}=-\frac{i}{2}\left(\hat{a}^{\dagger} \hat{b}-\hat{a} \hat{b}^{\dagger}\right) .
\end{aligned}
$$

For an $N$-photon state, the mathematical properties of these operators are identical to the spin operators for a spin quantum number of $j=N / 2$. It is thus possible to illustrate the possibility of enhancing the phase sensitivity geometrically by a three dimensional vector $\hat{\mathbf{J}}$, as shown in fig. 2. In particular, it is possible to represent the phase shift $\phi$ in the interferometer as a rotation of the vector $\hat{\mathbf{J}}$ around the $\hat{J}_{3}$ axis. The output

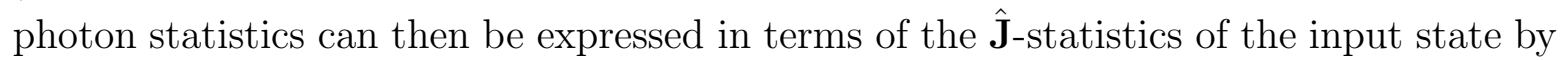
transforming the output operator $\hat{J}_{2}$, so that

$$
\begin{aligned}
\hat{J}_{2}(\phi) & =\exp \left(i \phi \hat{J}_{3}\right) \hat{J}_{2} \exp \left(-i \phi \hat{J}_{3}\right) \\
& =\cos (\phi) \hat{J}_{2}(0)+\sin (\phi) \hat{J}_{1}(0) .
\end{aligned}
$$

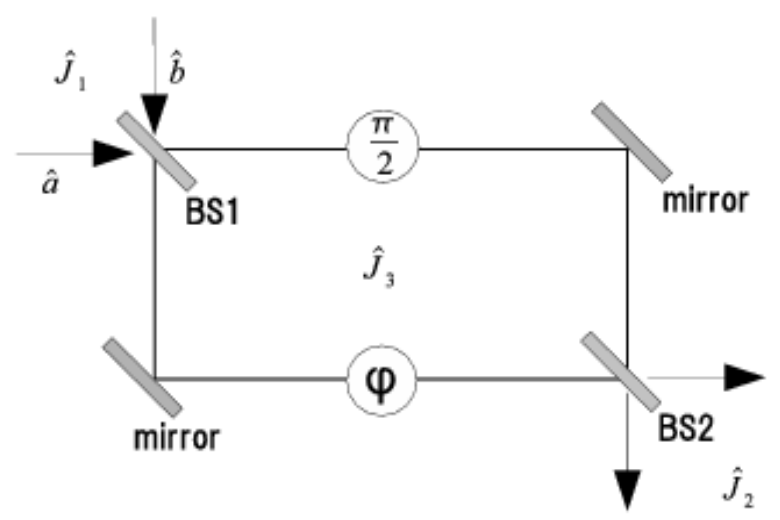

Figure 1. Illustration of the quantum mechanics of interference in a Mach-Zehnder interferometer. Light enters the interferometer in the input modes $\hat{a}$ and $\hat{b}$. The phase difference between the two paths inside the interferometer is $\phi-\pi / 2$. The photon statistics can be described by a three dimensional vector $\hat{\mathbf{J}}$, where $J_{1}, J_{2}$ and $J_{3}$ correspond to half of the photon number differences between the input modes, between the two paths inside the interferometer, and between the output modes at $\phi=0$, respectively. 

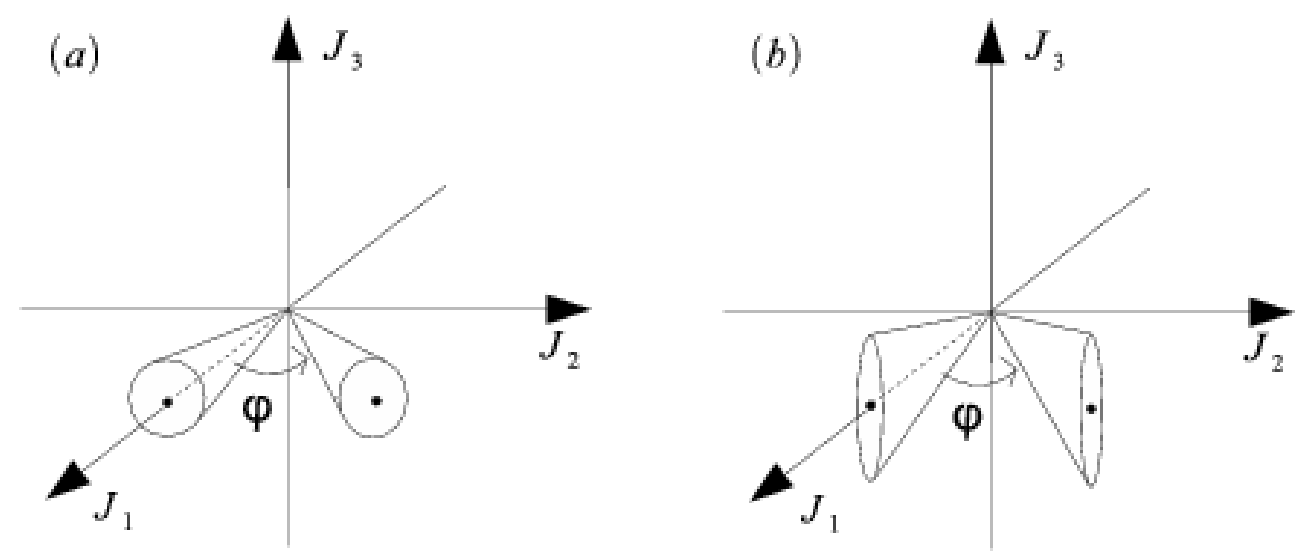

Figure 2. Illustration the effects of phase shifts $\phi$ on quantum states using the vector $\hat{\mathbf{J}}$ of the Schwinger representation. (a) shows the $|N ; 0\rangle$ state, generated by laser light in mode $\hat{a}$ and vacuum in mode $\hat{b}$. Since the $\hat{J}_{1}$ eigenvalue is maximal, the state is pointing in the $\hat{J}_{1}$ direction, surrounded by quantum noise equally distributed between $\hat{J}_{2}$ and $\hat{J}_{3}$. (b) shows an $N$-photon squeezed state with reduced fluctuations in $\hat{J}_{2}$ and correspondingly increased fluctuations in $\hat{J}_{3}$.

This relation expresses the complete dependence of the observable $\hat{J}_{2}$ measured in the output of the Mach-Zehnder interferometer on the phase $\phi$.

The dependence of the output statistics of $\hat{J}_{2}$ on the phase shift $\phi$ can be used to estimate the value of $\phi$ from the measurement results. For small phase shifts, the change of $\hat{J}_{2}$ is proportional to $\phi$, so a particularly intuitive phase estimate is obtained by dividing the measurement result of $\hat{J}_{2}$ by the phase derivative of the expectation value of $\hat{J}_{2}$. The sensitivity of this estimation procedure is limited by the uncertainty of the estimator observable $\hat{J}_{2}$ in the input of the interferometer. Specifically, the phase error $\delta \phi^{2}$ is given by the ratio of the squared $\hat{J}_{2}$-uncertainty $\Delta J_{2}^{2}$ and the squared phase derivative of the expectation value of $\hat{J}_{2}$,

$$
\delta \phi^{2}=\frac{\Delta J_{2}^{2}}{\left|\frac{\partial}{\partial \phi}\left\langle\hat{J}_{2}\right\rangle\right|^{2}}=\frac{\Delta J_{2}^{2}}{\left\langle\hat{J}_{1}\right\rangle^{2}} .
$$

The phase sensitivity of the input state can thus be improved by reducing the uncertainty in $\hat{J}_{2}$ while maintaining a high expectation value $\left\langle\hat{J}_{1}\right\rangle$.

To determine the kind of quantum states that are particularly suitable for the phase estimation procedure described above, it is useful to take a closer look at the Hilbert space geometry describing the uncertainty limit to the phase sensitivity given by eq.(3)). Assuming that $\left\langle\hat{J}_{2}\right\rangle=0$, the squared $\hat{J}_{2}$-uncertainty of an arbitrary input state $|\psi\rangle$ can be written as

$$
\Delta J_{2}^{2}=\left\langle\psi\left|\hat{J}_{2}^{2}\right| \psi\right\rangle=\left\langle\psi\left|\hat{J}_{2} \hat{J}_{2}\right| \psi\right\rangle .
$$

Therefore the squared uncertainty $\Delta J_{2}^{2}$ is the inner product of the uncertainty vector $\hat{J}_{2}|\psi\rangle$ with itself and the $J_{2}$-uncertainty $\Delta J_{2}$ is equal to the length $\| \hat{J}_{2}|\psi\rangle \|$ of this Hilbert space vector. On the other hand, the phase derivative of the expectation value 
of $\hat{J}_{2}$ can be expressed in terms of the commutation relation of the generator $\hat{J}_{3}$ and the estimator $\hat{J}_{2}$,

$$
\frac{\partial}{\partial \phi}\left\langle\hat{J}_{2}\right\rangle=\left\langle\hat{J}_{1}\right\rangle=-i\left(\left\langle\psi\left|\hat{J}_{2} \hat{J}_{3}\right| \psi\right\rangle-\left\langle\psi\left|\hat{J}_{3} \hat{J}_{2}\right| \psi\right\rangle\right) .
$$

That is, the phase derivative of $\left\langle\hat{J}_{2}\right\rangle$ is equal to twice the imaginary part of the inner product of $\hat{J}_{2}|\psi\rangle$ and $\hat{J}_{3}|\psi\rangle$. For $\left\langle\hat{J}_{2}\right\rangle=0$ and $\left\langle\hat{J}_{3}\right\rangle=0$, the lengths of these two Hilbert space vectors are given by the uncertainties $\Delta J_{2}$ and $\Delta J_{3}$. Since the inner product of two vectors cannot be larger than the product of the lengths of the two vectors, the uncertainty product is limited by the Cauchy-Schwartz inequality

$$
\Delta J_{2} \Delta J_{3} \geq \frac{1}{2} \frac{\partial}{\partial \phi}\left\langle\hat{J}_{2}\right\rangle .
$$

This is just a specific case of the Mandelstam-Tamm uncertainty relations that limit the phase sensitivities of quantum states [32]. A quantum state that achieves the limit of this uncertainty relation achieves its optimal phase sensitivity - the quantum CramerRao bound [1, 33] - with $\hat{J}_{2}$ as its optimal phase estimator. By substituting eq.(3) into eq.(6), it can be confirmed that the phase sensitivity limit is indeed equal to the well known bound given by the uncertainty of the generator $\hat{J}_{3}$,

$$
\delta \phi^{2} \geq \frac{1}{4 \Delta J_{3}^{2}} .
$$

The implications of this relation for optical quantum metrology have been explained with great clarity in [1]. In particular, eq.(7) shows that the maximal phase sensitivity is obtained for path entangled states, which have a maximal possible $\hat{J}_{3}$-uncertainty of $\Delta J_{3}^{2}=N^{2} / 4$ and can therefore achieve the Heisenberg limit (HL) of $\delta \phi^{2}=1 / N^{2}$. On the other hand, uncorrelated photons have a $\hat{J}_{3}$-uncertainty of $\Delta J_{3}^{2}=N / 4$, corresponding to a completely random distribution between the two paths of the interferometer. Nonentangled photons can therefore only achieve the standard quantum limit (SQL) of $\delta \phi^{2}=1 / N$.

Having established the consistency of our analysis with more general quantum metrology, we can now return to our specific phase estimation strategy characterized by the uncertainty vectors $\hat{J}_{2}|\psi\rangle$ and $\hat{J}_{3}|\psi\rangle$. According to eq.(55), the phase sensitivity of the estimator observable $\hat{J}_{2}$ is optimal if the vectors $\hat{J}_{2}|\psi\rangle$ and $\hat{J}_{3}|\psi\rangle$ have the same direction in Hilbert space with a purely imaginary inner product. If the ratio of the uncertainties in $\hat{J}_{2}$ and $\hat{J}_{3}$ is given by a squeezing factor of $\exp (-2 r)$, this condition can be expressed by

$$
\mathrm{e}^{r} \hat{J}_{2}|\psi\rangle=i \mathrm{e}^{-r} \hat{J}_{3}|\psi\rangle
$$

Eq.(8) defines the complete class of $N$-photon squeezed states for which $\hat{J}_{2}$ is the optimal phase estimator. Specifically, these states are all minimal uncertainty states of the $\hat{\mathbf{J}}_{\text {-vector components }} \hat{J}_{2}$ and $\hat{J}_{3}$, where the $\hat{J}_{2}$-uncertainty is squeezed and the $\hat{J}_{3^{-}}$ uncertainty is anti-squeezed, as illustrated in fig. 3 [26. Since the uncertainty product 


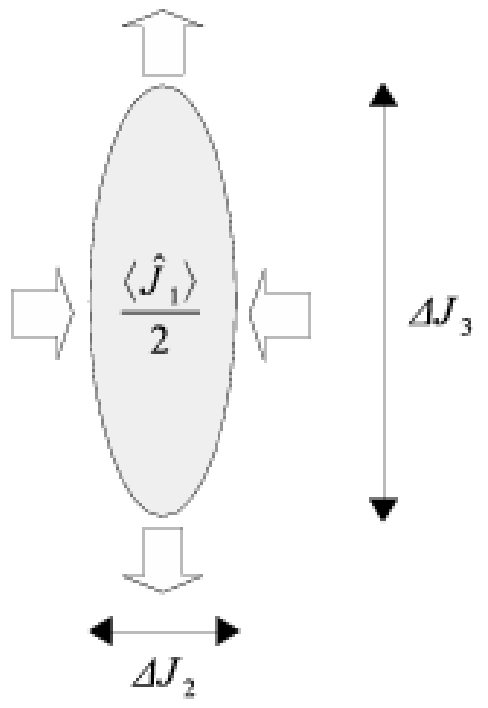

Figure 3. Illustration of squeezing in the $J_{2^{-}} J_{3}$ plane. A reduction of the output uncertainty $\Delta J_{2}$ requires an increase in path uncertainty $\Delta J_{3}$, so that the uncertainty product remains equal to $\left\langle\hat{J}_{1}\right\rangle / 2$.

$\Delta J_{2} \Delta J_{3}$ for these states is equal to its minimal value of $\left\langle\hat{J}_{1}\right\rangle / 2$, the uncertainties can be given separately as

$$
\Delta J_{2}^{2}=\frac{\mathrm{e}^{-2 r}}{2}\left\langle\hat{J}_{1}\right\rangle, \quad \Delta J_{3}^{2}=\frac{\mathrm{e}^{2 r}}{2}\left\langle\hat{J}_{1}\right\rangle .
$$

According to eq.(3) , the phase sensitivity of these states is then defined by the minimal phase error allowed by the uncertainty relation given by eq.(7),

$$
\delta \phi^{2}=\frac{\mathrm{e}^{-2 r}}{\left\langle\hat{J}_{1}\right\rangle} .
$$

Since this phase error cannot be lower than the HL of $1 / N^{2}$, we can expect that $\left\langle J_{1}\right\rangle$ will drop to zero with $\exp (-2 r)$. On the other hand, at squeezing levels well below the HL, we can expect $\left\langle J_{1}\right\rangle$ to be very close to $N / 2$, so that squeezing factors of $\exp (-2 r) \gg 1 / N$ directly describe the suppression of the phase error.

In the completely unsqueezed limit of $r=0$, eq.(8) actually defines the $\hat{J}_{1}$ eigenstate $|N ; 0\rangle$ with the maximal eigenvalue of $N / 2$, where all photons enter the Mach-Zehnder interferometer in mode $\hat{a}$. Experimentally, this state is easily realized by using coherent laser light to generate the single mode input in $\hat{a}$, while mode $\hat{b}$ is left in the vacuum state. The uncertainty vectors of this "classical" limit of quantum metrology are given by

$$
\hat{J}_{2}|N ; 0\rangle=\sqrt{\frac{N}{4}}|N-1 ; 1\rangle=i \hat{J}_{3}|N ; 0\rangle .
$$

Therefore, the uncertainties in $\hat{J}_{2}$ and $\hat{J}_{3}$ are both equal to $\sqrt{N / 4}$, which corresponds to the shot noise expected from a random distribution of the $N$ input photons between the paths and the output ports. The phase error of this "classical" input light is then given 
by the SQL of $\delta \phi^{2}=1 / N$, defining the phase sensitivity limit of completely uncorrelated photons.

In the following, we will look into the possibility of generating $N$-photon squeezed light that beats the SQL by quantum interference of laser light with only a small fraction of down-converted light. The resulting phase sensitivity will then be somewhere between the SQL and the HL. To evaluate just how strong the non-classical effects are, it is convenient to define the quantum enhancement parameter $Q$ with

$$
Q=\frac{\ln \left(1 /\left(N \delta \phi^{2}\right)\right)}{\ln (N)} .
$$

This parameter describes the logarithmic phase sensitivity on a scale from 0 for the SQL to 1 for the HL and thus provides a convenient tool for comparing the quantum enhancements achieved at different photon numbers $N$.

\section{Generation of $N$-photon squeezed states by quantum interference}

As explained in the previous section, the phase estimation error $\delta \phi^{2}$ originates from the uncertainty in the output photon number difference $\hat{J}_{2}$. In Hilbert space, this uncertainty

is described by a vector $\hat{J}_{2}|\psi\rangle$. It is therefore possible to reduce the uncertainty in $\hat{J}_{2}$ by adding a quantum state component whose uncertainty vector points in the opposite direction in Hilbert space. The uncertainties of the two components then interfer destructively, resulting in an $N$-photon squeezed state with a $\hat{J}_{2}$-uncertainty that is lower than that of either one of the components.

For the $|N ; 0\rangle$ state describing the laser input in mode $\hat{a}$, the uncertainty vector is given by

$$
\hat{J}_{2}|N ; 0\rangle=\sqrt{\frac{N}{4}}|N-1 ; 1\rangle .
$$

To reduce the uncertainty by quantum interference, we need an orthogonal state with an uncertainty component of $|N-1 ; 1\rangle$. Since the application of $\hat{J}_{2}$ exchanges exactly one photon between the input modes $\hat{a}$ and $\hat{b}$, the $|N-2 ; 2\rangle$ state satisfies this requirement. Specifically,

$$
\hat{J}_{2}|N-2 ; 2\rangle=\sqrt{\frac{N-1}{2}}|N-1 ; 1\rangle+\sqrt{\frac{3(N-2)}{4}}|N-3 ; 3\rangle .
$$

The $|N-1 ; 1\rangle$-component of this uncertainty vector has the same sign as the uncertainty in eq. (13), so a negative superposition of the shot noise limited laser light input $|N ; 0\rangle$ and the state $|N-2 ; 2\rangle$ with two photons in mode $\hat{b}$ will result in a reduction of the $\hat{J}_{2}$ uncertainty to below the SQL.

As has been shown in a number of papers [10, 12, 31, quantum interference effects can be obtained by combining weak coherent laser light with down-converted photon pairs. Just as in other down-conversion based experiments, the $N$-photon component is then selected by selectively detecting the $N$-photon coincidences in the output ports. In the present case, a quantum coherent superposition of the $|N ; 0\rangle$ state and the $|N-2 ; 2\rangle$ 
can be realized by adding weak parametric down-conversion (PDC) in mode $\hat{b}$ to the coherent laser light in mode $\hat{a}$. If the emission probability of photon pairs from PDC is very small, the down-converted light is approximately represented by the addition of a very small photon pair component to the vacuum state,

$$
|\gamma\rangle \approx|0\rangle-\frac{\gamma}{\sqrt{2}}|2\rangle
$$

where $\gamma \ll 1$. The remaining $N$ or $N-2$ photons originate from the coherent laser light in mode $\hat{a}$. The relevant components of the coherent state are given by

$$
|\alpha\rangle=\mathrm{e}^{-\frac{|\alpha|^{2}}{2}}\left(\ldots+\frac{\alpha^{N-2}}{\sqrt{(N-2) !}}|N-2\rangle \ldots+\frac{\alpha^{N}}{\sqrt{N !}}|N\rangle \ldots\right) .
$$

We can then obtain the $N$-photon component of the product state of eq.(15) and eq.(16),

$$
|\psi\rangle \approx|N ; 0\rangle-\sqrt{\frac{N(N-1)}{2}} \frac{\gamma}{\alpha^{2}}|N-2 ; 2\rangle,
$$

where the probability amplitude of $\sqrt{N(N-1) / 2}\left(\gamma / \alpha^{2}\right) \approx\left(N \gamma / \alpha^{2}\right) / \sqrt{2}$ should be much smaller than one. Note that the phase of the superpostion is controlled by the phase relation between the down-conversion amplitude $\gamma$ and the squared coherent state amplitude $\alpha^{2}$. Quantum interference between the two light sources is possible because the origin of the photons cannot be distinguished in the output measurements. The effect of this quantum interference on the uncertainty of the output photon number difference $\hat{J}_{2}$ is given by

$$
\Delta J_{2}^{2} \approx\left\langle N ; 0\left|\hat{J}_{2}^{2}\right| N ; 0\right\rangle-2 \sqrt{\frac{N(N-1)}{2}} \frac{\gamma}{\alpha^{2}}\left\langle N-2 ; 2\left|\hat{J}_{2}^{2}\right| N ; 0\right\rangle .
$$

The precise amount of noise reduction in the output is determined by the quantum interference term $\left\langle N-2 ; 2\left|\hat{J}_{2}^{2}\right| N ; 0\right\rangle$ of the operator $\hat{J}_{2}^{2}$. Using the appropriate matrix elements of $\hat{J}_{2}$, the result is

$$
\Delta J_{2}^{2} \approx \frac{N}{4}\left(1-2(N-1) \frac{\gamma}{\alpha^{2}}\right) .
$$

Since the change in $\left\langle\hat{J}_{1}\right\rangle$ is only of the order of $\gamma^{2}$, this reduction in the uncertainty of $\hat{J}_{2}$ translates directly into a corresponding reduction of the phase error. The quantum enhancement parameter $Q$ is then given by

$$
Q \approx 2 \frac{(N-1)}{\ln (N)} \frac{\gamma}{\alpha^{2}}
$$

Significantly, the quantum enhancement increases with the total photon number $N$, indicating that the selection of the $N$-photon component in the output plays an important role in the achievement of high squeezing levels. 


\section{Characterization of highly squeezed $N$-photon states}

In the previous section, we explained the basic effect of squeezing by quantum interference in the limit of very weak down-conversion, where the generation of multiple photon pairs can be neglected. However, the effect of multiple photon pairs in the downconverted light may be important for the generation of highly squeezed light. In this section, we therefore derive a more general expression for the interferometric squeezing effect based on the relation of the uncertainty vectors of $\hat{J}_{2}$ and $\hat{J}_{3}$ given by eq.(8) in section 2.

In general, the quantum state $|\gamma\rangle$ describing down-converted light is a slightly squeezed vacuum state. It is therefore possible to describe it as right eigenstate of a squeezed annihilation operator $\left(\hat{b}+\gamma \hat{b}^{\dagger}\right)$ with an eigenvalue of zero. Note that the parameter $\gamma$ describing the quadrature squeezing is usually very close to zero, since the quadrature squeezing achieved in conventional down-conversion is negligibly small. In the present context, the quadrature squeezing relation is used to characterize the quantum coherence of the down-converted light, which is described by the operator relation

$$
\hat{b}|\gamma\rangle=-\gamma \hat{b}^{\dagger}|\gamma\rangle .
$$

Likewise, the coherent state $|\alpha\rangle$ can be defined as the right eigenstate of its annihilation operator $\hat{a}$ with eigenvalue $\alpha$,

$$
\hat{a}|\alpha\rangle=\alpha|\alpha\rangle .
$$

We can now combine these two relations to characterize the coherence of the product state input $|\alpha ; \gamma\rangle$. However, this state is still a superposition of various total photon numbers $N$. Since we are interested in the results of coincidence counting experiments that select only the $N$-photon component of this state, where $N$ is usually much higher than the average photon number, it is necessary to separate the coherence of the $\mathrm{N}$ photon component from the (generally different) quantum coherences at other photon numbers. We have found that this problem can be solved by formulating an operator relation using only operators that do not change the total photon number, such as $\hat{a}^{\dagger} \hat{b}$ and $\hat{a} \hat{b}^{\dagger}$. The most simple relation we can thus derive is

$$
\begin{aligned}
\hat{a}^{\dagger} \hat{b}|\eta\rangle & =-\frac{\gamma}{\alpha^{2}} \hat{a}^{\dagger} \hat{a} \hat{a} \hat{b}^{\dagger}|\eta\rangle \\
& =-\eta \frac{\left(N-\hat{b}^{\dagger} \hat{b}\right)}{N} \hat{a} \hat{b}^{\dagger}|\eta\rangle,
\end{aligned}
$$

where $|\eta\rangle$ is the $N$-photon component detected in a corresponding coincidence counting experiment. The single parameter $\eta$ that defines the properties of this $N$-photon squeezed state is given by

$$
\eta=\frac{N \gamma}{\alpha^{2}} .
$$

Here, the definition of $\eta$ has been chosen so that the form of eq.(23) defining the $N$ photon state $|\eta\rangle$ is similar to the definition of the squeezed vacuum in eq.(21). As a 
result, the $N$-dependence of $\eta$ properly expresses the fact that the squeezing levels of the $N$-photon components in the same input state $|\alpha ; \gamma\rangle$ increase with $N$.

Using the Schwinger representation, we can now express the coherence operators $\hat{a}^{\dagger} \hat{b}$ and $\hat{a} \hat{b}^{\dagger}$ in terms of the output operator $\hat{J}_{2}$ and the path operator $\hat{J}_{3}$. We then obtain a non-linear version of the squeezing relation given by eq.(8),

$$
\left(1+\frac{\left(N-\hat{b}^{\dagger} \hat{b}\right)}{N} \eta\right) \hat{J}_{2}|\eta\rangle=i\left(1-\frac{\left(N-\hat{b}^{\dagger} \hat{b}\right)}{N} \eta\right) \hat{J}_{3}|\eta\rangle .
$$

Eq.(25) is an exact definition of the $N$-photon states generated by the interference of coherent laser light and down-converted light. However, the non-linear dependence of the quantum state on the photon number $\hat{b}^{\dagger} \hat{b}$ in the down-converted input beam introduces features that are quite different from the squeezing effects described in section 2 as $\eta$ increases [34]. In the following, we will therefore focus on the case of small $\eta$, where most of the photons originate from the coherent laser light input. We can then assume that $\left\langle\hat{b}^{+} \hat{b}\right\rangle \ll N$, so that the approximate equation for the $N$-photon squeezed state $|\eta\rangle$ can be written as

$$
(1+\eta) \hat{J}_{2}|\eta\rangle \approx i(1-\eta) \hat{J}_{3}|\eta\rangle .
$$

Except for a constant factor, this equation is equal to eq.(8)), and therefore describes the minimal uncertainty state introduced in section 2 with a squeezing factor of

$$
\mathrm{e}^{-2 r}=\frac{\Delta J_{2}}{\Delta J_{3}}=\frac{1-\eta}{1+\eta} .
$$

Thus, the $N$-dependent parameter $\eta=N \gamma / \alpha^{2}$ directly defines the level of squeezing for the $N$-photon component in the limit where most of the $N$ photons originate from the coherent light input. In particular, the $N$-dependence of the squeezing parameter

$\eta$ means that the squeezing properties of the input light are amplified by selecting a component with a photon number $N$ that is much greater than the average photon number of the input state, as it is presently done in typical down-conversion based experiments [8, 9, 11, 31]. Our result thus shows how the selection of $N$ photons in coincidence counting can result in strong non-classical effects, even though the actual squeezing level of down-converted light is negligible $(\gamma \ll 1)$.

\section{Limits of interferometric squeezing}

Eq.(27) suggests that arbitrarily high squeezing levels can be obtained as $\eta$ approaches one. However, it is clear that the approximation that most photons in the $\mathrm{N}$-photon state $|\eta\rangle$ originate from the coherent light input breaks down well before $\eta$ reaches one. It is therefore interesting to consider the limits of the approximation in order to determine the maximal squeezing levels that can be obtained by quantum interference between coherent light and down-converted light.

Since the approximation used to derive eq.(26) is based on the assumption that the number of down-converted photons in the $N$-photon state $|\eta\rangle$ is negligibly small, the limit of this approximation can be found by determining the approximate relation 
between the squeezing parameter $\eta$ and the average photon number in mode $\hat{b}$. To do so,

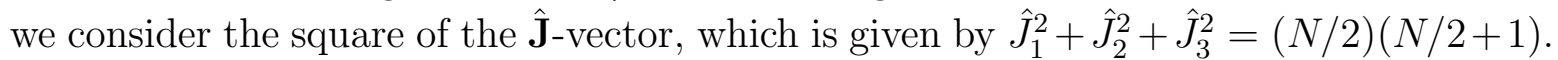
Since $\hat{J}_{1}=N / 2-\hat{b}^{\dagger} \hat{b}$, we can use the approximation $\left\langle\hat{J}_{1}^{2}\right\rangle \approx(N / 2)^{2}-N\left\langle\hat{b}^{\dagger} \hat{b}\right\rangle$ to derive the relation

$$
\left\langle\hat{J}_{2}^{2}\right\rangle+\left\langle\hat{J}_{3}^{2}\right\rangle \approx N\left(\left\langle\hat{b}^{\dagger} \hat{b}\right\rangle+\frac{1}{2}\right) .
$$

The expectation values $\left\langle\hat{J}_{2}^{2}\right\rangle$ and $\left\langle\hat{J}_{3}^{2}\right\rangle$ are equal to the uncertainties given by eq.(9), with $\left\langle\hat{J}_{1}\right\rangle \approx N / 2$ and $\exp (-2 r)=(1-\eta) /(1+\eta)$. The approximate relation between the squeezing parameter $\eta$ and the average photon number in input mode $\hat{b}$ thus reads

$$
\left\langle\hat{b}^{\dagger} \hat{b}\right\rangle \approx \frac{\eta^{2}}{1-\eta^{2}} .
$$

For $\eta \ll 1$, the average photon number in mode $\hat{b}$ increases with $\eta^{2}$, as expected from the approximate result in eq.(17) of section 3. Large photon numbers are only obtained when $\eta$ is close to one. We can therefore estimate that eq.(26) is valid until $(1-\eta)$ is considerably smaller than one.

It is now possible to get a more precise idea of where the deviations between the approximate definition of $|\eta\rangle$ in eq.(26) and the exact definition in eq.(25) become relevant. Specifically, the factor of $\left(1-\eta+\eta \hat{b}^{\dagger} \hat{b} / N\right)$ in the right hand side of eq.(25) is approximated by $(1-\eta)$ in the right hand side of eq.(26). Thus, the assumption is that

$$
1-\eta>\frac{\eta}{N}\left\langle\hat{b}^{\dagger} \hat{b}\right\rangle \text {. }
$$

For $(1-\eta) \ll 1$, the factor of $\eta$ on the right hand side of this relation is approximately equal to one. The same approximation can also be used to simplify the expression for the average photon number from down-conversion given by eq. (29) to $\left\langle\hat{b}^{\dagger} \hat{b}\right\rangle \approx 1 /(2(1-\eta))$. It is then possible to give the condition for the approximation used in eq.(26) as

$$
1-\eta>\frac{1}{\sqrt{2 N}}
$$

We can use this condition to derive estimates of the maximal average number of downconverted photons and of the minimal phase error achieved at the point where the approximation breaks down from eqs.(29) and (27). The results read

$$
\begin{aligned}
& \left\langle\hat{b}^{\dagger} \hat{b}\right\rangle<\sqrt{\frac{N}{2}}, \\
& \delta \phi^{2}>\frac{1}{(2 N)^{3 / 2}} .
\end{aligned}
$$

Eq.(32) indicates that the optimal squeezing levels will be achieved when the average photon number in mode $\hat{b}$ is close to $\sqrt{N / 2}$. At high photon numbers $N$, this is still only a small fraction of the total photon number. We can therefore conclude that most photons originate from the coherent laser light, even when the squeezing is maximal.

Eq.(33) provides an estimate of the amount of phase squeezing that can be achieved. Since it has been obtained by extrapolating the approximation of eq.(26) to the point where it breaks down, we can assume that the actual minimum of the phase error is a 
little bit higher than the lower bound given by eq.(26). In fact, numerical simulations such as the one presented in section 7 indicate that it is reasonable to expect the actual minimum phase error around $\delta \phi^{2}=1 / N^{3 / 2}$, which is equal to the geometric mean of the HL and the SQL. In terms of the quantum enhancement factor, the limit of phase sensitivity achieved by interferometric squeezing is given by

$$
Q_{\max } \leq \frac{1}{2} .
$$

The interference of laser light and down-converted photon pairs thus produces $N$-photon squeezed states with quantum enhancement factors of $Q=0$ to $Q=1 / 2$ for values of $\eta=0$ to $\eta=1$.

It may be worth noting that this result corresponds to the maximal phase sensitivity obtained by using interference between a squeezed vacuum and coherent light without selecting a specific $N$-photon component [28]. In that case, $N$ gives only the average photon number and the squeezing level is given directly by $\gamma$ instead of $\eta$. Thus, it is not immediately obvious that this result can also be applied to the actual $N$-photon component $|\eta\rangle$. However, it is possible to understand the similarity with our result by noting that, for average values of $N$ much greater than one, the photon numbers with the highest probabilities occur close to $N \approx \alpha^{2}$, where the $N$-photon squeezing parameter is $\eta \approx \gamma$. Thus the average phase sensitivity over all $N$ is close to the phase sensitivity observed for the special case of $N \approx \alpha^{2}$. By focussing on the quantum statistics of a specific $N$-photon component, our theory shows that the squeezing limit of $\delta \phi^{2}=1 / N^{3 / 2}$ applies not only to a high average photon number of $N \gg 1$, but also to the high $N$ components of extremely weak non-classical light observed by coincidence counting, where the squeezing parameter is increased by a factor of $N / \alpha^{2} \gg 1$.

Since our theory provides a more detailed description of the $N$-photon component, it also allows us to identify the kind of $N$-photon states generated at $\eta>1$. As we have shown elsewhere [34, the state generated at $\eta=2$ is actually close to a path entangled state, with a fidelity of $94 \%$ at high photon numbers $N$. We can therefore conjecture that eq.(25) describes a transition from squeezing to a quantum superposition

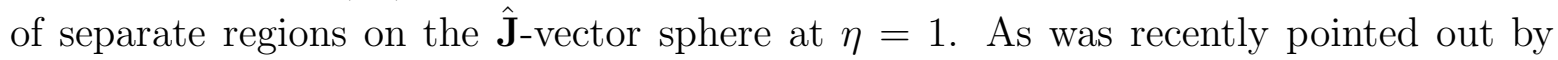
Pezze and Smerzi [35], it is possible to achieve the quantum Cramer-Rao bound for such states (and thus the Heisenberg limit at $\eta=2$ ) by optimizing the phase estimation method used to evaluate the output of the two path interferometer. This means that the phase sensitivity of the states actually continues to increase for $\eta>1$. However, the linear phase estimation procedure characterized by eq.(3) will not be optimal anymore, so that the high phase sensitivity can only be observed by using a more elaborate and error-sensitive phase estimation procedure.

\section{Generation probability of the $N$-photon $\eta$-state}

The two main advantages of the $N$-photon squeezed state $|\eta\rangle$ obtained by interference of laser light and down-converted photon pairs are that it permits a fairly straightforward 
and error-resistant phase estimation, and that it can be generated at high efficiency since most of the photons originate from the laser light input. In order to analyze the latter point, it is necessary to consider the complete quantum statistics of the interference between laser light and down-converted photon pairs. Since the photon number expansions of both the coherent state $|\alpha\rangle$ and the squeezed vacuum $|\gamma\rangle$ are well known, it is a straightforward matter to write out the complete product state in the input basis. The result is a superposition of outputs with different photon numbers $N$ given by

$$
|\alpha ; \gamma\rangle=\left(1-\gamma^{2}\right)^{1 / 4} \mathrm{e}^{-|\alpha|^{2} / 2} \sum_{N=0}^{\infty} \frac{\alpha^{N}}{\sqrt{N !}} \sum_{k=0}^{N / 2} \frac{1}{k !} \sqrt{\frac{(2 k) ! N !}{(N-2 k) !}}\left(\frac{\gamma}{2 \alpha^{2}}\right)^{k}|N-2 k, 2 k\rangle .
$$

The total probability of obtaining an $N$-photon squeezed state is then given by

$$
\begin{aligned}
& P_{\text {sq. }}(N)=\sqrt{1-\gamma^{2}} \mathrm{e}^{-|\alpha|^{2}} \frac{|\alpha|^{2 N}}{N !} \frac{1}{\left|C_{N}\right|^{2}}, \\
& \text { where } \quad \frac{1}{\left|C_{N}\right|^{2}}=\sum_{k=0}^{N / 2} \frac{(2 k) ! N !}{(k !)^{2}(N-2 k) !}\left|\frac{\gamma}{2 \alpha^{2}}\right|^{2 k} .
\end{aligned}
$$

The constant $C_{N}$ can also be used to express the normalized $N$-photon state $|\eta\rangle_{N}$, which is the state defined by eq.(25) with $\eta=N \gamma / \alpha^{2}$,

$$
|\eta\rangle_{N}=C_{N} \sum_{k=0}^{N / 2} \frac{1}{k !} \sqrt{\frac{(2 k) ! N !}{(N-2 k) !}}\left(\frac{\eta}{2 N}\right)^{k}|N-2 k ; 2 k\rangle .
$$

Here, $C_{N}$ is defined as the probability amplitude of the $|N ; 0\rangle$ component of the $N$ photon $\eta$-state. It may be worth noting that $C_{N}$ depends only on the value of the squeezing parameter $\eta$ and the total photon number $N$, so it can be determined without knowing the precise values of $\alpha$ and $\gamma$.

In previous experiments investigating the enhanced phase sensitivity of $N$-photon states, down-conversion was the only photon source used [9, 11, 20, 21, 22]. As a result, the $N$-photon coincidence count rate was limited by the available down-conversion amplitude $\gamma$, which is usually much smaller than one. In the case of interferometric squeezing discussed in this paper, the source of the non-classicality is also a downconversion process of amplitude $\gamma$. However, most of the $N$-photons of the squeezed states originate from the coherent laser light. It is therefore possible to achieve high $N$ photon coincidence rates, even if the down-converted amplitude $\gamma$ used is rather small. Since the technological requirements of interferometric squeezing are otherwise very similar to those for $N$-photon down-conversion, it is interesting to compare the $N$-photon squeezed state generation probability with the corresponding generation probability of an $N$-photon pair state $|N / 2 ; N / 2\rangle$ such as the one used in the recent demonstrations of phase super-resolution [21, 22].

When the down-conversion amplitude $\gamma$ is much smaller than one, the approximate generation probability for a pair state is given by

$$
P_{\text {pair }}(N)=\left(1-\gamma^{2}\right) \gamma^{N} \approx \gamma^{N}
$$


Table 1. Comparison of $N$-photon squeezed state generation probabilities $P_{\text {sq. }}$ with the generation probabilities $P_{\text {pair }}$ for $N$-photon pair states using the same downconversion source for photon number from $\mathrm{N}=3$ to $\mathrm{N}=8$. The three columns of the table show the improvement of coincidence count rates expected for $\eta=1 / 3, \eta=1 / 2$ and $\eta=1$ respectively. The numbers in parenthesis show the suppression of phase error below the standard quantum limit.

\begin{tabular}{c|cc|cc|cc|} 
& \multicolumn{2}{|c|}{$\eta=1 / 3$} & \multicolumn{2}{c|}{$\eta=1 / 2$} & \multicolumn{2}{c|}{$\eta=1$} \\
$N$ & $P_{\text {sq. }} / P_{\text {pair }}$ & $\left(N \delta \phi^{2}\right)$ & $P_{\text {sq. }} / P_{\text {pair }}$ & $\left(N \delta \phi^{2}\right)$ & $P_{\text {sq. }} / P_{\text {pair }}$ & $\left(N \delta \phi^{2}\right)$ \\
\hline 3 & 130 & $(0.68)$ & 40 & $(0.60)$ & 6 & $(0.75)$ \\
4 & $0.9 \times 10^{3}$ & $(0.64)$ & $1.9 \times 10^{2}$ & $(0.55)$ & 15 & $(0.57)$ \\
5 & $0.7 \times 10^{4}$ & $(0.62)$ & $0.9 \times 10^{3}$ & $(0.51)$ & 39 & $(0.51)$ \\
6 & $0.5 \times 10^{5}$ & $(0.60)$ & $0.5 \times 10^{4}$ & $(0.49)$ & 100 & $(0.45)$ \\
7 & $3.8 \times 10^{5}$ & $(0.58)$ & $2.4 \times 10^{4}$ & $(0.47)$ & 260 & $(0.41)$ \\
8 & $2.9 \times 10^{6}$ & $(0.57)$ & $1.2 \times 10^{5}$ & $(0.45)$ & 680 & $(0.37)$
\end{tabular}

Thus, the multi-photon generation probability falls off exponentially with $N$, indicating that the low down-conversion amplitude severely limits the efficiency of multi-photon pair state generation. The corresponding $N$-photon generation probability of an interferometric squeezed state is given by eq.(36). For a fixed down-conversion amplitude $\gamma$, it is possible to achieve any value of the squeezing parameter $\eta$, simply by varying $\alpha$ according to eq.(24). Specifically, the coherent amplitude needed to obtain a given value of the squeezing parameter $\eta$ is $\alpha=\sqrt{N \gamma / \eta}$. Using the assumption that both $\alpha$ and $\gamma$ are much smaller than one, the approximate $N$-photon generation probability then reads

$$
P_{\text {sq. }}(N) \approx \frac{1}{N !}\left(\frac{N \gamma}{\eta}\right)^{N} \frac{1}{\left|C_{N}\right|^{2}} \approx \frac{1}{\sqrt{2 \pi N}}\left(\frac{\mathrm{e} \gamma}{\eta}\right)^{N} \frac{1}{\left|C_{N}\right|^{2}}
$$

where the second approximation is made using the Sterling formula $N ! \approx \sqrt{2 \pi N} \mathrm{e}^{-N} N^{N}$ for large $N$. Eq.(39) shows that the $\eta$-state generation probability also falls off exponentially with photon number $N$. However, the exponential decline of the $\eta$-state coincidence count rate is slower than $N$-photon down-conversion by a factor of e/ $\eta$ in the base. The ratio of the $N$ photon generation probabilities of the squeezed state in eq.(36) and of the pair state in eq.(38) is therefore independent of $\gamma$ and reads

$$
\frac{P_{\text {sq. }}(N)}{P_{\text {pair }}(N)} \approx \frac{1}{\sqrt{2 \pi N}}\left(\frac{\mathrm{e}}{\eta}\right)^{N} \frac{1}{\left|C_{N}\right|^{2}} .
$$

Thus, the relative increase of the coincidence count rate compared to pair states is approximately exponential with photon number, indicating that interferometric squeezing is an especially promising method for increasing the number of photons $\mathrm{N}$ observed in coincidence counting.

To illustrate the improvement in the coincidence count rate over pair state generation, table 1 shows the ratio $P_{\text {sq. }} / P_{\text {pair }}$ for the experimentally relevant range of 
photon numbers from 3 to 8 photons. The ratios have been determined from eq.(36) and eq.(38) without any approximations. Of particular interest might be the case of $N=4$, since four photon coincidences have been used in the recent experiments demonstrating phase super-sensitivity [21, 22]. In this case, beating the SQL with a highly squeezed four photon state at $\eta=1$ would already improve the coincidence count rate by a factor of 15 . By reducing the squeezing parameter $\eta$ to $1 / 3$, the count rate can even be increased to 900 times that of pair state generation, at a phase error that is still lower than the SQL by a factor of 0.64. In general, the results in table 1 show that the coincidence count rates of interferometric squeezed states should be several orders of magnitude higher than those obtained in pair state generation, even at presently accessible photon numbers.

\section{Numerical results for the eight photon squeezed state}

To confirm the validity of the approximations used in the previous sections and to take a closer look at the maximally squeezed state, we now investigate the specific numerical results that can be obtained for the $N=8$ case. According to eq.(37), the eight photon state is given by

$$
\begin{aligned}
|\eta\rangle_{N=8} & =C_{8}\left(|8 ; 0\rangle-\frac{\sqrt{7}}{4} \eta|6 ; 2\rangle+\frac{3 \sqrt{70}}{64} \eta^{2}|4 ; 4\rangle-\frac{15 \sqrt{7}}{256} \eta^{3}|2 ; 6\rangle+\frac{105}{4096} \eta^{4}|0 ; 8\rangle\right) \\
& \approx C_{8}\left(|8 ; 0\rangle-0.661 \eta|6 ; 2\rangle+0.392 \eta^{2}|4 ; 4\rangle-0.155 \eta^{3}|2 ; 6\rangle+0.0256 \eta^{4}|0 ; 8\rangle\right) .
\end{aligned}
$$

In this expansion, the first two terms correspond exactly to the $N=8$ case of the approximation given in eq.(17). Below $\eta=1 / 2$, these two terms alone contribute more than $99 \%$ of the state. Above $\eta=1 / 2$, higher order terms become relevant and the approximations for low $\eta$ cease to apply.

We can now determine the phase error $\delta \phi^{2}$ and the quantum enhancement parameter $Q$ from the expectation values of the eight photon state according to eq.(3) and eq.(12). The result is shown in fig. 4, together with the rough approximation of the $\eta$-dependence of squeezing given by eq.(27). The validity of this approximation is restricted to very low values of $\eta$, since the assumption that the average photon number in mode $\hat{b}$ is much smaller than $N$ breaks down very quickly at only eight photons. In fact, the approximation bends upward at all values of $\eta$, while the actual quantum enhancement curve bends downward. As a result, the values of $\eta$ necessary to achieve a given squeezing factor are actually higher than those expected from eq.(27). For example, a squeezing factor of $1 / 2$ corresponding to $Q=1 / 3$ is only reached at $\eta=0.427$ instead of $\eta=1 / 3$. On the other hand, the approximate estimate of the maximal value of $Q=1 / 2$ given by eq.(34) is confirmed by the maximum of $Q=0.51$ at $\eta=0.85$. At eight photons, this corresponds to an optimal squeezing factor of 0.346 times SQL, only slightly better than the rough estimate of $1 / N^{3 / 2}=0.354$ corresponding to $Q=1 / 2$. 


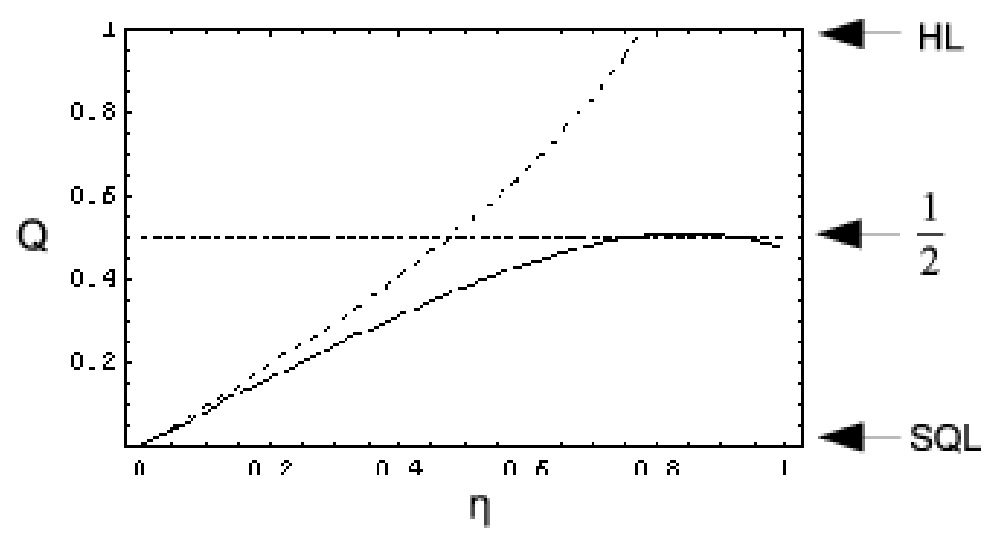

Figure 4. Quantum enhancement parameter $Q$ describing the phase sensitivity achieved by the eight photon state as a function of $\eta$. The dashed line shows the approximate value of $Q$ corresponding to a squeezing factor of $\exp (-2 r)=$ $(1-\eta) /(1+\eta)$.

Since eq.(41) defines the values of the normalization coefficient $C_{8}$, it is also possible to determine the improvements of coincidence rates compared to the generation of an eight photon pair state $|4 ; 4\rangle$ more precisely. For example, the state at $\eta=0.427$, which has a phase error equal to $1 / 2$ of the SQL, has a normalization factor of $\left|C_{8}\right|^{2}=0.922$. From eq.(40), we can then determine that the coincidence rate will be $4.1 \times 10^{5}$ times higher than the coincidence rate of pure down-conversion. At the optimal squeezing level of 0.346 times SQL, we have $\eta=0.85$ and $\left|C_{8}\right|^{2}=0.712$. According to eq.(40), the coincidence rate is then about 2200 times higher than that of pure down-conversion. It should be noted that this result is significantly higher than the factor of 680 estimated for $\eta=1$ in table 1. In general, the improvements in coincidence count rates are quite significant and should be an important help in the realization of non-classical multiphoton states.

Next, we take a closer look at the kind of state described by the eight photon output given in eq.(41). In section 4, we have shown that the state is approximately equal to a minimal uncertainty state of the Mandelstam-Tamm inequality (7) for the $\hat{J}_{2}$ estimator, so that the phase error defined by eq. (3) should be equal to the optimal phase sensitivity of $1 /\left(4 \Delta J_{3}^{2}\right)$. As a test of this approximation, we can now compare the actual values of the phase error $\delta \phi^{2}$ and the optimal phase sensitivity of $1 /\left(4 \Delta J_{3}^{2}\right)$ obtained from the expectation values of the actual eight photon state. The result of this comparison is shown in fig. 5. Interestingly, the phase error $\delta \phi^{2}$ is indeed close to its optimal value of $1 /\left(4 \Delta J_{3}^{2}\right)$ up to about $\eta=0.5$. This means that the state is very close to a minimal uncertainty state defined according to eq.(8), even though the relation between $\exp (-2 r)$ and $\eta$ given by eq.(27) seems to break down at much lower values of $\eta$.

At $\eta>1 / 2$, the optimal phase sensitivity of $1 /\left(4 \Delta J_{3}^{2}\right)$ continues to drop, but the phase error $\delta \phi^{2}$ for the $\hat{J}_{2}$ estimator levels off and reaches a minimum of about 0.043 


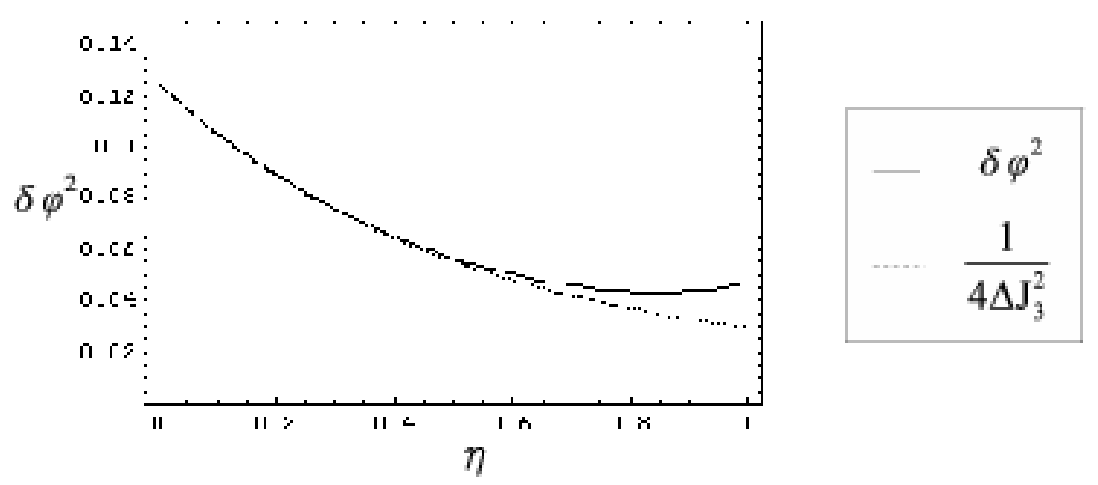

Figure 5. Comparison of the phase error $\delta \phi^{2}$ and the optimal phase sensitivity of $1 /\left(4 \Delta J_{3}^{2}\right)$ for the eight photon state. Up to $\eta=1 / 2$, the state is an optimally squeezed state. Above $\eta=1 / 2, \delta \phi^{2}$ reaches a minimum value of 0.043 at $\eta=0.845$, while $1 /\left(4 \Delta J_{3}^{2}\right)$ continues to drop towards the HL of 0.0156 .

at $\eta=0.845$. At higher $\eta$, the $\hat{J}_{2}$ based estimate actually becomes worse, even though the potential phase sensitivity indicated by the generator uncertainty $\Delta J_{3}^{2}$ continues to improve. This means that the state at $\eta>1 / 2$ requires a different estimation procedure to achieve its optimal phase sensitivity of $1 /\left(4 \Delta J_{3}^{2}\right)$ [35]. As we have shown elsewhere, the state eventually evolves into a state that is very close to the maximally path entangled state at $\eta=2$ [34]. Above $\eta=0.5$, the eight photon state thus makes a transition from a squeezed state with an optimal phase estimator of $\hat{J}_{2}$ to a superposition state with an optimal phase estimator that must take into account multi-photon coherences between the paths.

We can thus see that the quantum interferometric squeezed state naturally combines aspects of multi-photon interference fringes with the classical dependence of the output photon number difference $\hat{J}_{2}$ on the phase shift $\phi$ in the interferometer. Since the relation between the measurement probabilities themselves and the $\phi$ dependence of $\hat{J}_{2}$ given by eq.(2) may not be immediately apparent, we conclude our numerical analysis with an illustration of the phase dependence of the complete output photon number distribution. Fig. 6 (a) shows the photon statistics of the unsqueezed state at $\eta=0$, and fig. 6 (b) shows the corresponding statistics at $\eta=0.85$, close to the maximally squeezed state. The graphs on the left hand side show the $\hat{J}_{1}$ distribution and the $\hat{J}_{2}$ distribution, while the graphs on the right handside show a contour plot of the phase dependence of the output photon number distributions defined by the eigenstates of $\hat{J}_{2}(\phi)$ given by eq.(2). The shading along each line represents the probability of a specific measurement outcome. An artificial discretization of phase into 20 intervals of $\pi / 10$ has been used to simplify the plot. We can see from the graphs on the right hand side that the peak of the probability distribution follows the sine-pattern expected from classical interference. However, the unsqueezed state (a) is rather broad at $\phi=0, \pi, 2 \pi$ and sharp at $\phi=\pi / 2$ and $3 \pi / 2$, while the squeezed state (b) is sharper at $\phi=0, \pi, 2 \pi$ and shows periodic oscillations within a broadened $\hat{J}_{2}(\phi)$ distribution at $\phi=\pi / 2$ and $3 \pi / 2$. It is thus possible to visualize both the classical aspects of field amplitude interference and the 

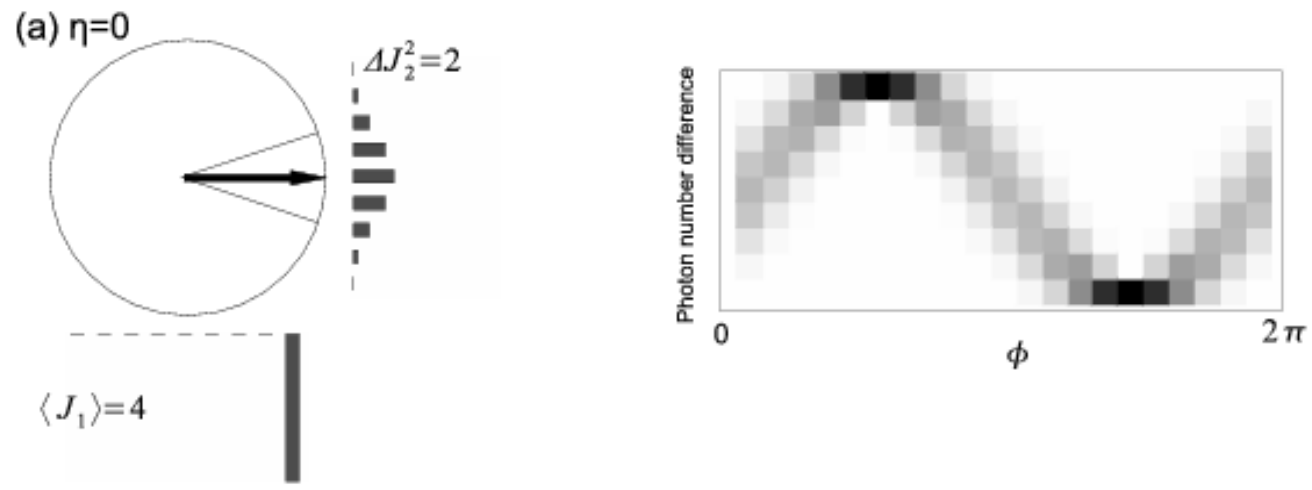

(b) $\eta=0.85$
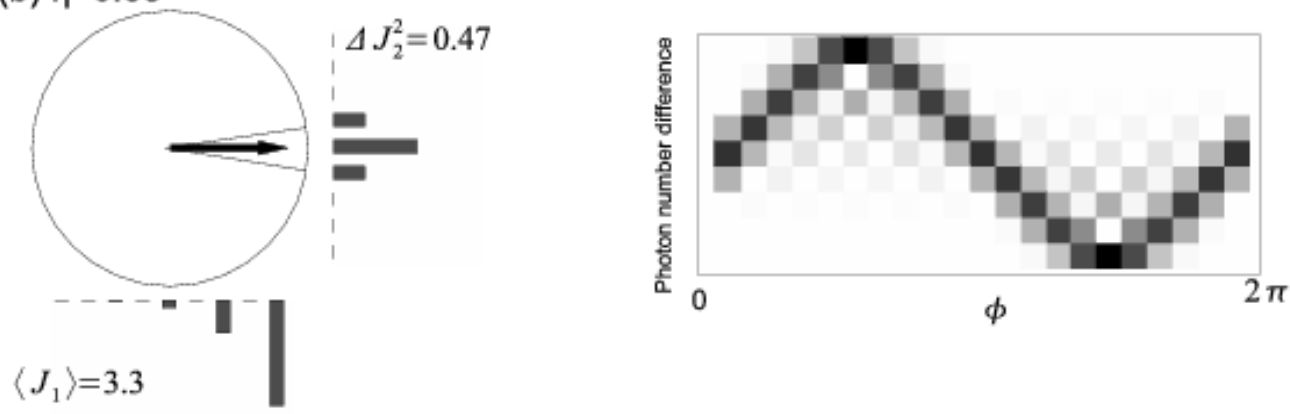

Figure 6. Illustration of the quantum statistics of eight photon states for (a) the unsqueezed state with $\eta=0$ and (b) the highly squeezed state at $\eta=0.85$. The graphs on the left show the $\hat{J}_{1}$ and $\hat{J}_{2}$ distributions of the input and the output at $\phi=0$. The graphs on the right show the phase dependence of the probability distributions as a contour plot. The high probability regions indicated by the darker shadings reproduce the classical sinusoidal dependence of the output photon number difference $\hat{J}_{2}(\phi)$.

quantum aspects of probability amplitude interferences in the same graph by plotting the phase dependent many photon distribution of the quantum state.

\section{Conclusions}

In conclusion, we have shown how the interferometric addition of down-converted photon pairs to weak coherent light can produce $N$-photon squeezed states that are minimum uncertainty states of the standard phase estimation based on the output photon number difference $\hat{J}_{2}$. Significantly, the amount of squeezing depends only on the ratio of $N$ times the down-conversion amplitude $\gamma$ and the squared coherent amplitude $\alpha$. In typical down-conversion experiments using coincidence counting, $N / \alpha^{2} \gg 1$ allows an enhancement of the negligibly small downconversion amplitude $\gamma \ll 1$ to the very high squeezing levels described by $\eta$ close to one. It is therefore possible to obtain very high squeezing levels, even at very low down-conversion rates.

In our derivations, we have tried to emphasize the rather intriguing relation between measurement noise and quantum interference in Hilbert space. It is thus possible to directly understand squeezing as a destructive interference between the quantum noise 
terms originating from the laser light and from the down-conversion, as discussed in section 3. However, the same effect can also be represented in terms of an operator relation that defines the minimal uncertainty of the Mandelstam-Tamm uncertainty relation, as explained in section 2. Since we can derive the general operator relation (25) for the $N$ photon state $|\eta\rangle$ generated by interference of laser light and downconverted light, it is possible to identify the squeezing effect even more directly based on an approximate version of this operator relation, as shows in section 4 .

Our results indicate that we can obtain any squeezing level between the SQL and the geometric mean of the SQL and the HL by varying the ratio of the down-conversion amplitude and the squared coherent light amplitude. For a given level of photon pair emission in the down-conversion, any desired squeezing can be obtained by varying the laser amplitude. As explained in section 6, the coincidence count rates for these phase sensitive $N$-photon states can then be orders of magnitude higher than those observed for pure down-conversion. In particular, there is a trade-off between the coincidence count rates and the amount of squeezing that can be adjusted to fit the experimental possibilities. The generation of $N$-photon squeezed states by interfering coherent light and down-converted light at a beam splitter should thus be ideally suited for beating the SQL of quantum metrology at increasing photon numbers $N$.

On a more fundamental level, the present approach should also help to improve our understanding of quantum metrology by bridging the gap between the $N$ photon interference effects of path entangled states and the continuous improvement of phase sensitivity by squeezing. We therefore hope that the present work will open up new frontiers in the study of quantum phase measurements.

\section{Acknowledgment}

Part of this work has been supported by the Grant-in-Aid program of the Japanese Society for the Advancement of Science and by the JST-CREST project on quantum information processing.

[1] Giovannetti V, Lloyd S and Maccone L 2006 Phys. Rev. Lett. 96010401

[2] Edamatsu K, Shimizu R and Itoh T 2002 Phys. Rev. Lett. 89213601

[3] Kok P, Lee H and Dowling J P 2002 Phys. Rev. A 65052104

[4] Fiurasek J 2002 Phys. Rev. A 65053818

[5] Zou X, Pahlke K and Mathis W 2002 Phys. Rev. A 66014102

[6] Pryde G J and White A G 2003 Phys. Rev. A 68052315

[7] Hofmann H F 2004 Phys. Rev. A 70023812

[8] Walther P, Pan J W, Aspelmeyer M, Ursin R, Gasparoni S and Zeilinger A 2004 Nature (London) 429158

[9] Mitchell M W, Lundeen J S and Steinberg A M 2004 Nature (London) 429161

[10] Shafiei F, Srinivasan P and Ou Z Y 2004 Phys. Rev. A 70043803

[11] Eisenberg H S, Hodelin J F, Khoury G and Bouwmeester D 2005 Phys. Rev. Lett. 94090502

[12] Liu B and Ou Z Y 2006 Phys. Rev. A 74035802

[13] Nielsen A E B and Molmer K 2007 Phys. Rev. A 75063803

[14] Cable H and Dowling J P 2007 Phys. Rev. Lett. 99163604 
[15] Resch K J, Pregnell K L, Prevedel R, Gilchrist A, Pryde G J,O'Brien J L and White A G 2007 Phys. Rev. Lett. 98223601

[16] Holland M J and Burnett K 1993 Phys. Rev. Lett. 711355

[17] Steuernagel O 2002 Phys. Rev. A 65033820

[18] Wang H and Kobayashi T 2005 Phys. Rev. A 71 021802(R)

[19] Hofmann H F 2006 Phys. Rev. A 74013808

[20] Sun F W, Liu B H, Huang Y F, Ou Z Y and Guo G C 2006 Phys. Rev. A 74033812

[21] Nagata T, Okamoto R, O‘Brien J, Sasaki K and Takeuchi S 2007 Science 316726

[22] Sun F W, Liu B H, Gong Y X, Huang Y F, Ou Z Y and Guo G C 2007 Preprint arXiv:0710.2922 1

[23] Caves C M 1981 Phys. Rev. D 231693

[24] Yurke B, McCall S L and Klauder J R 1986 Phys. Rev. A 334033

[25] Kitagawa M and Ueda M 1993 Phys. Rev. A 47, 5138

[26] Hillery M and Mlodinow L 1993 Phys. Rev. A 481548

[27] Wineland D J, Bollinger J J, Itano W M and Heinzen D J 1994 Phys. Rev. A 5067

[28] Paris M G A 1995 Phys. Lett. A 201132

[29] Berry D W and Wiseman H M 2000 Phys. Rev. Lett. 855098

[30] Combes J and Wiseman H M 2005 J. Opt. B: Quantum and Semiclass. Opt. 714

[31] Lu Y J and Ou Z Y 2001 Phys. Rev. Lett. 88023601

[32] Braunstein S L, Caves C M and Milburn G J 1996 Ann. Phys. 247135

[33] Braunstein S L and Caves C M 1994 Phys. Rev. Lett. 723439

[34] Hofmann H F and Ono T 2007 Phys. Rev. A 76 031806(R)

[35] Pezze L and Smerzi A 2007 Preprint arXiv:0705.4631v1 